\title{
LA RELACIÓN ENTRE AUTONOMÍA Y VULNERABILIDAD EN HABITANTES DE CALLE: UN RETO PARA LA BIOÉTICA
}

\author{
Carolina Soto-Méndez ${ }^{1}$
}

Resumen: El propósito de este artículo es identificar las relaciones entre vulnerabilidad y autonomía que ha desarrollado la literatura académica en relación con los habitantes de calle. Para el cumplimiento del objetivo se desarrolló búsqueda bibliográfica en distintas bases de datos bajo los criterios de habitante de calle, autonomía, toma de decisiones, vulnerabilidad, bioética. Como conclusión es claro que los problemas mentales que pueden presentar aquel grupo poblacional evidencian las dificultades para dirimir la falsa dicotomía bioética entre autonomía y vulnerabilidad. Se identifica entonces una nueva tarea para la bioética, como es superar la dicotomía presentada que pueda aportar elementos sustanciales que ayuden a sobrepasar las dificultades para la atención de habitantes de calle.

Palabras clave: habitante de calle, autonomía, toma de decisiones, vulnerabilidad, bioética

\section{The relationship of homeless, autonomy and vulnerability: a challenge for bioethics}

\begin{abstract}
The purpose of this article is to identify the relationships between vulnerability and autonomy that academic literature has developed in relation to homeless. For the fulfillment of the objective, a bibliographic search was developed in different databases under the criteria of homeless, autonomy, decision making, vulnerability, bioethics. As a conclusion, it is clear that the mental problems that this population group can present demonstrate the difficulties that exist to resolve the false bioethical dichotomy that exists between autonomy and vulnerability. A new task for bioethics is identified, such as overcoming the presented dichotomy that can provide substantial elements that help to overcome the difficulties that arise for the attention of homeless.
\end{abstract}

Keywords: homeless, autonomy, vulnerability, decision making, bioethics

\section{A relaçáo entre autonomia e vulnerabilidade em moradores de rua: um desafio para a bioética}

Resumo: O objetivo deste artigo é identificar as relaçóes entre vulnerabilidade e autonomia elaboradas pela literatura acadêmica em relaçáo aos moradores de rua. Para alcançar este objetivo realizou-se uma pesquisa bibliográfica em diferentes bases de dados, utilizando-se como critérios morador de rua, autonomia, tomada de decisóes, vulnerabilidade, boiética. Como conclusão, ficou claro que os problemas mentais que aquele grupo populacional pode apresentar evidenciam as dificuldades que existem para dirimir a falsa dicotomia bioética entre autonomia e vulnerabilidade. Identifica-se, então, uma nova tarefa para a bioética, a de superar a dicotomia apresentada de forma a fornecer elementos substanciais que ajudem a superar as dificuldades que se apresentam para a atenção aos moradores de rua.

Palavras-chave: morador de rua, autonomia, tomada de decisóes, vulnerabilidade, boiética

\footnotetext{
${ }^{1}$ Grupo de Investigación Liderazgo, línea Bioética, Biopolítica y Biojurídica, Universidad Militar Nueva Granada, Colombia Correspondencia: carosotomendez@gmail.com
} 


\section{Introducción}

El fenómeno de los habitantes de la calle es una realidad social y, en ese sentido, son éstos sujetos de especial protección por parte del Estado colombiano $(1,2)$. De esta manera, se define "habitante de calle" como la persona (sin ninguna distinción) que hace de la calle su lugar permanente de habitación, de manera permanente o transitoria(2). La vinculación de un habitante de la calle a procesos de rehabilitación debe ser totalmente voluntaria(3), implica que debe entenderse la autonomía como elemento primordial en la toma de decisiones de rehabilitación. Sin embargo, la autonomía de los habitantes de calle debe interpretarse en relación de la vulnerabilidad de su condición, pues así se han reconocido $(2,4,5)$. De esta manera, se hace necesario comprender las dificultades que se originan en los procesos de toma de decisión en los habitantes de calle para su rehabilitación, de acuerdo al reconocimiento establecido en sentencias de la Corte Constitucional colombiana $(1,3)$. En este sentido, la bioética permite considerar elementos que faciliten la interpretación del proceso de toma de decisiones.

Por eso se plantea como objetivo de este artículo identificar las relaciones entre vulnerabilidad y autonomía que ha desarrollado la literatura académica en relación con los habitantes de calle. Para lograr lo propuesto, se presenta primero la metodología mediante la cual se hizo la revisión bibliográfica, y, luego, en los resultados, se indican los hallazgos bibliográficos a partir de la búsqueda en habitantes de calle, autonomía, vulnerabilidad y bioética, para finalmente, en la discusión, plantear las conexiones entre estos tres elementos.

\section{Metodología}

Para el desarrollo de este artículo de revisión bibliográfica, se realizó la búsqueda en bases de datos a partir del descubridor de la biblioteca de la Universidad Militar Nueva Granda, que localiza textos en las bases de datos de ElSevier, Ebsco, Dialnet, MedPlus, ScienceDirect, Redalyc, como también a partir de la búsqueda en Google Académico. El rastreo se concentró en los términos "habitantes de calle", "autonomía", "vulnerabilidad", "bioética" y "toma de decisiones", que fueron utilizados como criterios de palabras clave, título de los textos o texto completo. La combinación de todos los términos mencionados arrojaba como búsqueda sin resultados; entonces, se optó por la búsqueda combinada de máximo tres descriptores (bioética, habitantes de calle, autonomía; habitantes de calle, vulnerabilidad, toma de decisiones; habitantes de calle, vulnerabilidad, autonomía).

Una vez obtenidos los textos, se prefirió la inclusión de aquellos que desarrollaran la temática de los habitantes de calle adultos, sin decir que fueran excluidos totalmente los que desarrollaran de forma exclusiva la problemática de los niños habitantes de calle menores de edad. Se identificaron preferentemente documentos que estudiaran el fenómeno de habitantes de calle en Colombia o en América Latina, por las características propias que tiene el fenómeno en el territorio colombiano.

Los criterios de selección fueron textos que desarrollaran una comprensión del habitante de calle sin importar desde el área del conocimiento que se presentara, prefiriendo aquellos que hicieran un estudio más completo del fenómeno y no enfocándose solo en menores de edad. Una vez seleccionados, se procedió a registrar la información recopilada en una matriz de análisis que facilitara la comprensión de la situación de los habitantes de calle en relación con la autonomía y la vulnerabilidad.

\section{Resultados}

No siempre ha existido notorio interés en el estudio de los habitantes de calle adultos, pues ha existido mayor interés en el fenómeno cuando son menores de edad(6). Para su comprensión, ha existido necesidad de generar un concepto uniforme sobre habitante de calle, pero no ha sido posible porque varía de acuerdo al momento histórico $(7,8)$, pues no siempre se ha conocido con la misma denominación(9); al país(10) e incluso de la región continental(11), como también de la perspectiva en la que se enfoque. Es claro que no puede confundirse con conceptos como "indigente”, que tiene una connotación económica(5); sin embargo, son asumidos como sinónimos por los demás ciudadanos(12). 
Tampoco es lo mismo hablar de "habitante de calle" y "habitante en calle"; habitante de calle es aquel que hace de la calle su sitio permanente de vivienda y rompe los vínculos con su familia y habitante en calle es el menor de 18 años(5). Rodríguez(13), por su parte, hace la diferenciación desde la óptica de los planteamientos de la Secretaría de Integración Social, al considerar que habitante en calle es quien pasa la mayor parte de su tiempo en la calle sin hacer de ella su lugar de habitación permanente. Burbano(14), al momento de conceptualizar sobre el habitante de calle, además de los elementos mencionados, tiene en cuenta la vulnerabilidad de su condición.

Así, la vulnerabilidad surge como un elemento esencial en la condición del habitante de calle. Garspard y Rivera(15) consideran que es una condición que se encuentra implícita en el habitante de calle al establecer que "Drogadicción y errancia pueden conjugarse en situaciones donde se impone la desprotección y un desapego con respecto al propio cuerpo, que se acentúa con el consumo repetitivo de drogas"(15:329). Se identifican varios estudios que relacionan directamente la vulnerabilidad de la salud con la condición de habitar en calle $(4,14,16,17)$. Pero no es el único enfoque de vulnerabilidad que se considera en relación con los habitantes de calle; por ejemplo, Leão, Veiga y Passarella(18) muestran que la población anciana y en general los habitantes de la calle, tienen una condición de vulnerabilidad que conjuga la vulnerabilidad laboral, habitacional, entre otras. Rosa(19) expresa que "los habitantes de la calle viven en extrema vulnerabilidad; se encuentran excluidos del ámbito laboral, de la posibilidad de tener su vivienda, de una salud física y mental íntegra, etc." (19:192), aunque no se separen de la sociedad. Otra faceta de la vulnerabilidad, con la que es recurrente identificar a los habitantes de calle, es la vulnerabilidad social(19-22), pues en muchos casos las personas llegan a habitar en calle por situaciones de violencia intrafamiliar, maltrato, etc.

La vulnerabilidad hace parte de las formas como la sociedad reconoce a los habitantes de calle o como se reconocen a sí mismos; Tamayo y $\mathrm{Na}$ varro(24) exponen que las representaciones sociales son "formaciones cognitivas compartidas por un mismo grupo social" (24:7) y dejan ver que, en relación a su seguridad, los habitantes de calle perciben que la tienen (en aspectos generales), mientras los operadores públicos no lo consideran así, por los problemas de limpieza social. La percepción que tienen sobre los derechos humanos permite observar la apreciación que tienen los habitantes de calle sobre su condición de precariedad y la deshumanización que reciben(25). La sociedad los percibe como vulnerables $(8,12)$, porque además los lugares que frecuentan tienen una alta relación con fenómenos de inseguridad en las ciudades(26).

De otro lado, los habitantes de calle son percibidos como excluidos sociales $(4,5,24,27-30)$, pues hacen parte de un proceso de privación económica, social y política(29) en el que se refleja el modelo económico imperante de una $\operatorname{sociedad}(6)$. Sin embargo, no todos los sectores perciben al habitante de calle de esa manera, pues encuentran que se relacionan con otro estilo de vida, probablemente nómada(31), incluso pueden llegar a tener una autopercepción no de exclusión, sino de expresión de libertad(9).

Sobre los motivos que llevan a una persona a ser habitante de la calle, es claro que es una situación multifactorial, porque "el modo de vida en la calle se articula a condiciones objetivas y subjetivas de existencia que determinan principios generadores y organizadores de las prácticas de los individuos, es decir, determinan las posibilidades y los límites de las prácticas de los sujetos en su cotidianidad"(32:71); en Colombia, la Política Distrital de Habitabilidad en Calle de Bogotá, indica como causas del fenómeno el consumo de "sustancias psicoactivas diferentes al alcohol, el deterioro de la red familiar, la búsqueda de medios de subsistencia y otras problemáticas relacionadas con el ámbito familiar, como por ejemplo el abandono (sobre todo de personas mayores o con discapacidad), [...] y la búsqueda de medios de subsistencia"(33:16); sin embargo, sí se traduce el alcoholismo en una de las razones de permanencia en calle(33, p.39).

En la investigación que desarrollaron Ricardo, Correa, Velásquez, Gómez, Franco y Celis sobre las características sociodemográficas de niños y adolescentes de la calle, en Medellín, identificaron que el maltrato familiar es una causa principal 
de vivir en calle(34). En Colombia también está asociado al desplazamiento por el conflicto armado interno y a la pobreza extrema $(27,30)$; además, se reconoce que la causa de descomposición social y la ruptura de vínculos familiares y sociales están presentes en los habitantes de la calle(28).

Los trastornos mentales también están presentes en la población habitante de calle(16,30,34-36), entre los que se encuentran la drogadicción $(7,15,28)$ y el alcoholismo $(7,15,33)$, y se puede comprender que ambas sean situaciones que se prolonguen en el tiempo durante la condición de habitante de calle(15). No obstante, del habitante de calle se predica autonomía $(1,2)$ y en su cotidianidad se autopercibe libre por la forma en que se sujeta a las normas(37).

Es claro que la situación de los habitantes de calle se ha convertido en una problemática para los gobiernos locales (5,38). Arroyave(38) explica que este sector de la población es de especial interés para el Estado porque de ellos hacen parte los sin techo, merecedores de intervención estatal. Correa(5) manifiesta que las acciones que el Estado plantea como estrategia de desarrollo, con el fin de erradicar la pobreza y la exclusión, deben tener condiciones más específicas que faciliten un apoyo que permita prevenir condiciones tempranas de riesgo y vulnerabilidad.

Aunque está consagrado para los habitantes de calle, en la práctica existen diversas razones y limitantes por las cuales éstos no acceden a la atención en salud(29). De otro lado, las acciones de intervención en esta población se plantean debido a la necesidad de brindar herramientas que posibiliten su tratamiento en farmacodependencia $(6,39)$, pues el consumo de sustancias psicoactivas es una condición generalizada en los habitantes de ca$\operatorname{lle}(29,40)$.

Los programas para habitantes de calle buscan promover espacios de inclusión social a partir de garantizar los mínimos necesarios para su subsistencia(30). Sin embargo, el acceso a procesos de rehabilitación debe ser totalmente voluntario, pues se reconoce la autonomía del habitante de calle(1-3). Esto representa una situación compleja en Colombia, pues aunque la Corte Constitucional colombiana ha establecido el criterio de la autonomía en habitantes de calle(3), no tiene criterio único para la situación de quienes se necuentran en condición de discapacidad mental, situación agravada por la insuficiencia legislativa en materia de discapacidad en Colombia(41).

La deshospitalización de enfermos mentales produjo, más que la reducción de este fenómeno, el desplazamiento hacia otros espacios, como es la calle; cuando por situaciones de seguridad son desalojados de determinados sectores, son retornados nuevamente a la institución junto con otras personas y todos son reconocidos bajo la categoría de excluidos sociales. Desde la bioética, es una situación que puede ser reflexionada a la luz de la justicia distributiva(30).

Los habitantes de calle que son identificados como consumidores de sustancias psicoactivas ilegales están expuestos a una doble vulnerabilidad: por su condición de vida en la calle y por ser consumidores. $\mathrm{Y}$ es precisamente en ese aspecto que puede aportar la bioética, una comprensión de la doble vulnerabilidad en la que éstos se encuentran(28).

\section{Discusión}

El concepto de "habitante de calle" ha sido uno de los temas más estudiados por los investigadores(4-14); entonces, a pesar de que existan diferencias entre regiones o países $(10,11)$, no hay consenso básico y es una condición que va más allá de la falta de un lugar de habitación que tenga unas condiciones mínimas y básicas, pues en el habitante de calle se reconoce la no satisfacción de una u otra forma de las necesidades básicas de subsistencia de una persona. Ser habitante de calle, entonces, está mediado por la desprotección de los derechos de manera previa, pues cuestiones como el maltrato intrafamiliar, el consumo de psicoactivos, el desplazamiento forzoso interno y condiciones de extrema pobreza son situaciones previas a la configuración de la condición de habitante de calle.

Salta a la vista la forma recurrente que se hace uso del concepto "vulnerabilidad", bien para indicar sus condiciones de salud, como para indicar sus condiciones de vida en general. La conceptualización que se observa de "vulnerabilidad" es in- 
dicada para establecer un modelo predictivo, con el fin de medir la vulnerabilidad al VIH, como en el caso del estudio de Berbesi, Segura, Cardona y Caicedo(17). En este estudio parten de la definición de "vulnerabilidad" como de una situación generada por la ruptura de un equilibrio que pone énfasis en la incapacidad de adaptarse, resistirse y adaptarse a los fenómenos naturales. No se indaga por las formas de vulnerabilidad posibles en el habitante de calle, sino que parte del supuesto que todo habitante de calle está inmerso en una situación de vulnerabilidad por el simple hecho de su condición. Los estudios que buscan las condiciones de salud de la población habitante de calle $(4,8,11,14,16,17)$ indagan por la susceptibilidad de sufrir un daño, pero lo hacen desde la óptica de la epidemiología o desde la salud pública(8).

Ten Have(42) plantea que, desde las disciplinas científicas, se ha entendido la vulnerabilidad en sentido funcional, y explica que en los habitantes de calle (homeless) la vulnerabilidad es multidimensional y la habitabilidad de calle (homelessness) es el mejor ejemplo para explicar que la vulnerabilidad es un concepto relacional más que individual, porque una persona es siempre vulnerable a algo o a alguien. Además, explica que, por ser un concepto usado por varias disciplinas o áreas del conocimiento, existen múltiples interpretaciones y formulaciones para la vulnerabilidad; entonces, Ten Have(42) explica que la vulnerabilidad tiene un marco bioético, porque no puede limitarse solamente al aspecto médico ya que significa caer en la falsa dicotomía entre vulnerabilidad y autonomía.

El planteamiento de la multidimensional de la vulnerabilidad(42) concuerda con los estudios que han identificado diversas facetas de la vulnerabilidad en los habitantes de calle, como es en cuanto a las condiciones de salud $(4,14,16,17)$, social(19-22) y multifactorial. De esta manera, se identifica la necesidad de profundizar en la comprensión de la vulnerabilidad en los habitantes de calle desde una perspectiva bioética.

Sin embargo, el enfoque de vulnerabilidad desde la bioética clínica plantea que a mayor vulnerabilidad menor autonomía $(42,43)$. Esto se convierte en una situación conflictual, porque a los habitantes de calle, para el acceso a cualquier tratamiento de rehabilitación, se les reconoce su autonomía $(1-3,41)$ y sin su permiso no pueden ser internados en ninguna institución con tal fin. Además, como se ha observado ampliamente, son portadores de una condición vulnerable. El problema se complejiza frente a los habitantes de calle que tienen algún tipo de problema men$\operatorname{tal}(7,15,16,28,30,35,36)$, porque puede existir de manera subyacente una condición inhabilitante para el proceso de toma de decisión.

Sin embargo, el enfoque de la Convención sobre los Derechos de las Personas con Discapacidad reconoce la autonomía a este grupo poblacional(44) y la Corte Constitucional colombiana también ha tomado ese camino en relación a los habitantes de calle $(1,3)$. No obstante, no evidencia uniformidad en sus criterios respecto al respeto de la autonomía de personas con discapacidad(41).

La existencia de criterios para el reconocimiento de la autonomía en personas con discapacidad mental no es suficiente para superar la falsa dicotomía expuesta por Ten Have(42). Para Ribeiro y Flor do Nacimento es una situación que se puede superar si se abandona el abordaje del principialismo bioético norteamericano y en su lugar se instala en la bioética de la intervención(28), pues ésta tiene la capacidad de abordar los problemas sociales que impactan la vida y la salud, y de esta manera se puede superar la visión clínica para reflexionar acerca de las diversas formas de vulnerabilidad(28). Es importante aclarar que la bioética de la intervención es una de las perspectivas bioéticas que se gestan en Latinoamérica a la par de la bioética de protección(45-47) y la centrada en los derechos humanos(45). El enfoque de la bioética de intervención está centrado en la transformación de las prácticas sociales, con un alto compromiso social, pues en Latinoamérica no se puede dejar de lado discusiones sobre los sistemas de salud en cuanto a su funcionamiento, uso de recursos, entre otros(46).

El enfoque que plantea la bioética de la intervención hace propicio el cuestionamiento del fenómeno de la habitabilidad en calle desde una perspectiva de la vulnerabilidad(28); sin embargo, la propuesta que realizan Ribeiro y Flor do Nacimento se centra en considerar la vulnerabi- 
lidad y los derechos humanos de los sujetos consumidores de drogas y habitantes de calle, con el interés de superar una visión biomédica. No obstante, en el contexto colombiano, la discusión se centra en si un habitante de calle puede ser o no obligado a un proceso de rehabilitación, lo que requiere el concurso de perspectivas biomédicas de la bioética; significa entonces que el fenómeno de habitabilidad en calle puede abordarse desde otras orientaciones bioéticas, porque, entre otros, plantea un problema de salud pública(33) y conjuga claramente problemas de inequidad social; como lo explica Quintero, es un problema que también puede ser abordado desde la justicia distributiva(30).

El fenómeno de habitabilidad en calle plantea entonces la necesidad de profundizar en los aspectos que permitan, desde la bioética, una relación distinta entre autonomía y vulnerabilidad, que facilite el abordaje en habitantes de calle sin estar limitado por los planteamientos propios de un enfoque bioético, y que no sea solamente un tema que pueda ser abordado por una perspectiva bioética en particular, para así aportar posibles repuestas al problema del abordaje del habitante de calle en miras a una eventual rehabilitación.

\section{Conclusión}

El fenómeno de los habitantes de calle plantea un escenario claro de la relación autonomía-vulnerabilidad, debido a que las situaciones que llevan a alguien tomar la decisión de habitar en calle tienen un origen en diversas situaciones que generan desprotección de las personas, situación que se observa en la revisión de literatura realizada. Los habitantes de calle, por su condición, se encuentran en un estado de vulnerabilidad permanente, en un grado mayor que cualquier otra persona. Los problemas mentales que puede presentar aquel grupo poblacional evidencian las dificultades que existen para dirimir la falsa dicotomía bioética que existe entre autonomía y vulnerabilidad. Se identifica entonces una nueva tarea para la bioética, como es superar la dicotomía presentada que pueda aportar elementos sustanciales que ayuden a sobrepasar las dificultades que se presentan para la atención de habitantes de calle.

\section{Conflicto de intereses}

Los autores manifiestan que no existe conflicto de intereses al respecto.

\section{Referencias}

1. Corte Constitucional Colombiana. Sentencia T-057 de 2011. (M.P. Jorge Iván Palacio Palacio, 2011, 4 de febrero). Disponible en: http://www.corteconstitucional.gov.co/relatoria/2011/t-057-11.htm

2. Ley 1641 de 2013. (12 de julio) por la cual se establecen los lineamientos de la política pública social para habitantes de la calle y se dictan otras disposiciones. Disponible en: http://wsp.presidencia.gov.co/Normativa/Leyes/Documents/2013/LEY\%201641\%20DEL\%2012\%20DE\%20JULIO\%20DE\%202013.pdf

3. Corte Constitucional Colombiana. Sentencia T-043 de 2015. (M.P. Jorge Iván Palacio Palacio, 2015, 4 de febrero). Disponible en: http://www.corteconstitucional.gov.co/relatoria/2015/t-043-15.htm

4. Berbesy D, Agudelo A, Segura A, Montoya L. VIH en habitantes de Calle de Medellín. Rev. Fac. Nac. Salud Pública 2012; 30(3): 310-314.

5. Correa M. Para una nueva comprensión de las características y la atención social a los habitantes de la calle. Eleuthera 2007; 1: 91-102.

6. Correa M. La otra ciudad - otros sujetos: los habitantes de calle. Trabajo Social 2007; 9: 37-56.

7. Nieto C. Consumo de drogas en tres etapas de la vida de habitantes de calle de Bogotá: predictores de consumo y comparación con una muestra de población infantil y adolescente de Brasil. [Tesis de Maestría] Universidad Federal do Rio Grande do Sul. 2011. Disponible en: https://www.lume.ufrgs.br/handle/10183/37297

8. Daza L. Determinantes sociales del fenómeno de habitabilidad de calle en Bogotá D.C. Una aproximación desde la salud urbana. Medicina U.P.B. 2017; 36(1): 51 -58.

9. Suarez C. Estigma, communitas y modos de corrección para los habitantes de la calle en Bogotá (2000-2010). Sociedad y Economía 2016; 32: 195-216.

10. Nieto C, Koller S. Definiciones de Habitante de Calle y de Niño, Niña y Adolescente en Situación de Calle: Diferencias y Yuxtaposiciones. Acta de Investigación Psicológica 2015; 5(3): 2162-2181. 
11. Tipple G, Speak S. Definitions of homelessness in developing countries. Habitat International 2005; 29: 337-352.

12. Navarro O, Gaviria M. Representaciones sociales del habitante de la calle. Universitas Psychologica 2010; 9(2): 345-355.

13. Rodríguez C. Del cuerpo social al cuerpo femenino callejero: una mirada de las políticas sociales en Bogotá. Papel Político 2015; 20(1): 187-211.

14. Burbano Y. Evaluación de las acciones del programa de control y prevención de tuberculosis de la secretaría distrital de salud de Bogotá D.C. en el marco de la estrategia alto a la tuberculosis como parte de la atención integral al habitante de calle en el hogar uno día noche durante 2009 y 2010. [Tesis Maestría]. Pontificia Universidad Javeriana; 2012. Disponible en: https://repository.javeriana.edu.co/handle/10554/3839

15. Gaspard J, Rivera S. Drogadicción y aislamiento social. Reflexiones sobre la atención a drogadictos en Francia y Colombia. Avances en Psicología Latinoamericana 2016; 34(2): 315-338.

16. Correa M, Orozco M, Uribe M, Barraza T, Zapata A, Villa C, Correa C. Habitantes de calle y tuberculosis: una realidad social en Medellín. Eleuthera 2012; 6: 101-126.

17. Berbesi D, Segura A, Cardona D, Caicedo B. Índice de vulnerabilidad al VIH en población habitante de calle. Enfermería Global 2017; 46: 154-167.

18. Leâo F, Veiga M, Passarella A. Pobre, anciano y en la calle: una trayectoria de exclusión. Revista Latrinoamericana de Enfermagemm 2007; 15 (spe): 755-761.

19. Rosa C. "Excluido por excelencia": revisiones de un concepto para el caso de los habitantes de la calle. Revista de Estudios Regionales 2011; 7: 185-196.

20. León H. De la marginalidad a la inclusión social. Sistematización de una experiencia de formación y capacitación a través del trabajo productivo. [Tesis Maestría]. Convenio Universidad Pedagógica Nacional Centro Internacional De Educación Y Desarrollo Humano - Cinde; 2006. Disponible en: http://repository.cinde. org.co/handle/20.500.11907/1884

21. Mellizo H. La niñez habitante de la calle en Colombia reflexiones, debates y perspectivas. Tendencias y Retos 2005; 9(32).

22. Moxley D, Washington O. The Relevance of Four Narrative Themes for Understanding Vulnerability Among Homeless Older African-American Women. Practice social work in acction 2015; 27(2): 113-133.

23. Ossa L. (Adolescentes) en situación de calle: Construcción de identidad en situación de extrema vulnerabilidad. Un acercamiento cualitativo. [Tesis Maestría]. Universidad de Chile; 2005. Disponible en: http://repositorio. uchile.cl/handle/2250/106001

24. Tamayo W, Navarro O. Representación Social del habitante de calle. Revista Psicología 2009; 1(1): 7-34.

25. Ocampo S. El ser humano como ciudadano: una mirada desde los derechos humanos de los habitantes de y en la calle de Bogotá, localidad de Los Mártires. Criterio Jurídico Garantista 2016; 9(15): 86-101.

26. Filgado A, Suarez C, Vallejo E. Faces da ilegalidade em Bogotá. Tempo Social 2010; 22(2): 123-142.

27. Restrepo A. El ser humano al límite: una mirada reflexiva al habitante de la calle. Drugs and Addictive behavior 2016; 1(1): 89-100.

28. Ribeiro L, Flor do Nacimento W. Drogas, direitos humanos e bioética: dupla vulnerabilidade do usuário de drogas em situação de rua. Revista Latinoamericana de Bioética 2017; 17(2): 63-75.

29. Barbesy D, Agudelo L, Castaño C, Galeano M, Segura A, Montoya L. Utilización de los servicios de salud en la población habitante de calle. CES Salud Pública 2014; 5: 147-153.

30. Quintero L. La exclusión social en "habitantes de la calle" en Bogotá. Una mirada desde la bioética. Revista Colombiana de Bioética 2008; 3(1): 101-144.

31. Ruiz J. Los citadinos de la calle. Nómadas 1999; 10: 172-177.

32. Moreno C, Espinosa G, Zapata L. Entre el hogar y el asfalto: relatos y experiencia de vida de habitantes en condición de calle. Revista Lasallista de Investigación 2017; 14(2): 65-72.

33. Alcaldía Mayor de Bogotá. Decreto 560 de 2015. Política Pública Distrital para el Fenómeno de Habitabilidad en calle. Disponible en: http://old.integracionsocial.gov.co/anexos/documentos/2015politicaspublicas/1603 2017_Pol\%C3\%ADtica_P\%C3\%BAblica_Distrital_de_Habitabilidad_en_Calle_PPDFHC.pdf

34. Ricardo C, Correa M, Velasquez J, Gómez M, Franco J, Celis M. Características sociodemográficas y trastornos mentales en niños y adolescentes habitantes de la calle en un centro de atención social de Medellín, 
La relación entre autonomía y vulnerabilidad en habitantes de calle: un reto para la bioética - Carolina Soto-Méndez

Colombia. Medicina UPB 2011; 30(1): 21-29.

35. Klein J, Reddy S. Care of the Homeless Patient. Medical Clinics of North America 2015; 99: 1017-1038.

36. Ayano G, Assefa D, Haile K, Chaka A, Solomon H, Hagos P, Yohannis Z, Haile K, Bekana L, Agidew M, Demise S, Tsegaye B, Solomon M. Mental, neurologic, and substance use (MNS) disorders among street homeless people in Ethiopia. Ann Gen Psychiatry 2017; 16(40).

37. Betancur A, Castro D. Significados de las normas sociales y las figuras de autoridad en personas adultas con experiencia de vida en calle. Psicoespacios: Revista virtual de la Institución Universitaria de Envigado 2016; 10(16): 82-102.

38. Arroyave A. El problema público del habitante de calle en Medellin 2012-2015: un estudio desde el instrumento de regulación legal. [Artículo Maestría]. Universidad EAFIT; 2016. Disponible en: https://repository.eafit. edu.co/handle/10784/11321

39. Jaramillo T, Restrepo A, Restrepo S. Escritura emocional: una estrategia para la resocialización del habitante de calle adulto de Medellin. [Informe de Investigación]. Universidad de Manizales - CINDE; 2014. Disponible en: http://ridum.umanizales.edu.co:8080/xmlui/bitstream/handle/6789/1827/Restrepo_Sandra_Milena_2014.pdf?sequence $=1$ \&isAllowed $=y$

40. Hernández-Carrillo M, Álvarez-Claros K, Osorio-Sabogal I. Consumo autoreportado de sustancias psicoactivas ilegales en una población habitante de calle de Cali-Colombia. Rev. salud pública 2015; 17(2): 217-228.

41. Jaime J. La sentencia T-043 de 2015 de la Corte Constitucional de Colombia, la batalla de la capacidad legal en las calles de Medellín, Pereira y el Bronx. Revista Latinoamericana en Discapacidad, Sociedad y Derechos Humanos 2017; 1(1): 175-186.

42. Ten Have H. Vulnerability challenging bioethics. New York: Routledge Taylor \& Francis Group; 2016.

43. Rosas C. La vulnerabilidad humana ¿un freno a la autonomía?. Rev Bioet Latinoam. 2015; 16: 1-16.

44. Organización de las Naciones Unidas. Convención Sobre Los Derechos De Las Personas Con Discapacidad. Disponible en: http://www.un.org/esa/socdev/enable/documents/tccconvs.pdf..

45. Rivas-Muñoz F, Garrafa V, Ferreira S, Flor de Nascimento W. Bioética de intervención, interculturalidad y no-colonialidad. Saúde Soc. São Paulo 2015; 24(1): 141-151.

46. Arpini A. Para una fundamentación de la Bioética de Intervención. Aportes desde la Ética de la liberación latinoamericana. Rev. Redbioétical UNESCO 2016; 7-1(13): 12-23.

47. Ferreira S, Flor do Nascimento W. La bioética de intervención en el contexto del pensamiento latinoamericano contemporáneo. Rev. bioét. (Impr.). 2015; 23(2): 281-288. 